\title{
How fast can thiols bind to the gold-nanoparticle surface?
}

Amanda Bard, Rebeca Rondon, Daniela T. Marquez, Anabel E. Lanterna*, Juan C. Scaiano*

Department of Chemistry and Biomolecular Sciences, Centre for Advanced Materials Research (CAMaR), University of Ottawa, Ottawa, Ontario K1N 6N5, Canada

*Corresponding authors' e-mails: alantern@uottawa.ca (AEL) jscaiano@uottawa.ca (JCS)

\section{ORCID numbers:}

15 A.E. Lanterna: 0000-0002-6743-0940; J.C. Scaiano: 0000-0002-4838-7123

${ }^{a}$ Dedicated to Professor E.A. Lissi, as he reaches a new milestone. 


\begin{abstract}
20 Kinetics of gold-nanoparticle-surface modification with thiols can take more than one hour for completion. 7-Mercapto-4-methylcoumarin, can be used to follow the process by fluorescence spectroscopy and serves as a convenient molecular probe to determine relative kinetics of other thiol compounds. SERS studies with aromatic thiols further support the slow surface modification kinetics observed by fluorescence spectroscopy. The formation of thiolate bonds

25 is a relatively slow process; we recommend one to two hour wait for thiol binding to be essentially complete, while for disulfides, overnight incubation is suggested.
\end{abstract}




\section{INTRODUCTION}

While there are numerous ways to stabilize gold nanoparticles (AuNP) the most common ones

30 involve the formation of the thiolate $\mathrm{S}-\mathrm{Au}$ bond $(\sim 130 \mathrm{~kJ} / \mathrm{mol})$, frequently by reaction of thiols with the gold surface (1). The modification of gold surfaces with thiol compounds has been extensively explored as one of the best gold surface passivation methods, as well as the best way to anchor different functional groups to the surface (1-6). While the strategy has proven thermodynamically effective and efficient, the complex kinetics of the reaction have been

35 frequently overlooked. The process is well understood when it involves the formation of selfassembled monolayers (SAM) on atomically flat gold surfaces, but to a lesser extent in the case of AuNP where polydispersity and irregularities on the surface and curvature variations make the binding process more complex and the conclusions more difficult to generalize (7). Surface enhanced Raman spectroscopy (SERS) has proven a useful tool to study structural effects and

40 kinetics of thiol-AuNP interactions (7, 8), although relatively large particle sizes (e.g. 50-200 $\mathrm{nm}$ ) and aromatic thiols tend to be preferred to suit SERS optimal experimental conditions (9). Thus, we combine fluorescence and SERS as a way to generalize our results to different chemical structures and particle sizes.

While working on preparing thiolated stabilized AuNP, we have asked ourselves how long we

45 had to wait until the derivatization was complete, so as to avoid working with nanostructures that were still undergoing significant change. A few literature studies (such as those mentioned above) address this question for specific systems, yet, much more frequent are literature contributions where the delay between thiolate derivatization and usage of the nanostructures is simply not mentioned. A study of the influence of thiolate derivatization on the catalytic

50 reduction of 4-nitrophenol serves as a perfect example of the importance of thiolate coverage on 
the catalytic performance (10). The free catalytic site density determines the activity of the material. Interestingly, considerable catalytic activity is retained even when the coverage is 90 $\%$.

The motivation for this study is our interest in the delay between reagent mixing and completion 55 of the S-Au derivatization process. In order to address this issue, we employed two strategies. One of these methodologies involves simple fluorescence spectroscopy. In our work, fluorescence spectroscopy is used as a tool that is suitable for different combinations of nanostructure and sensing molecules (Scheme 1). In the other approach, Raman spectroscopy is used to further support our results. While recognizing that our data contain kinetic and

60 mechanistic information and do some simple kinetic analysis, we use a rather pragmatic approach by trying to answer this question. It is important to note that the type (particularly size) of nanoparticles and the organic structures required to optimize fluorescence and SERS measurements are different and thus the sections that follow, dealing with fluorescence and Raman spectroscopy are not directly comparable. In this case, this is an asset, as it allows us to

65 establish the generality of the conclusions we reach in this contribution. Scheme 1 shows the molecules examined in this contribution.

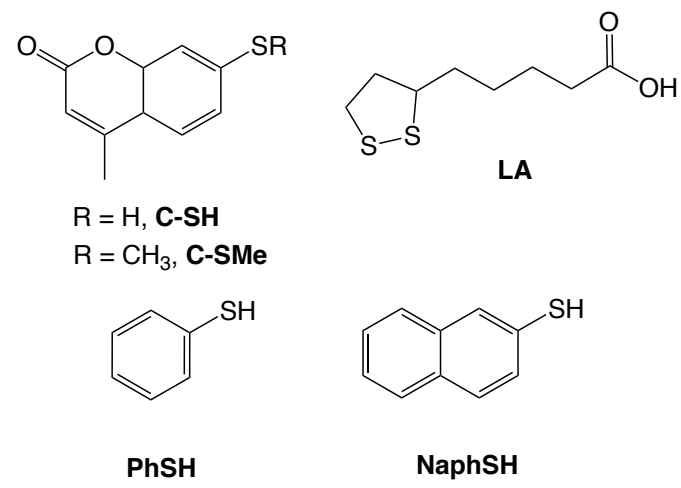

Scheme 1. Thiol compounds used in this study. 


\section{MATERIALS AND METHODS}

Materials and Instrumentation: All reagents have been purchased from Sigma-Aldrich and have been used without further purification unless otherwise stated. S-methyl-7-mercapto-4methylcoumarin (C-SMe) was synthesized as previously described (11). AuNPs were synthesized using a reported method (12) via reduction with sodium citrate. Briefly, $230 \mathrm{~mL}$ of

75 a $0.3 \mathrm{mM}$ aqueous solution of $\mathrm{HAuCl}_{4}$ were heated up to boiling point, then $20 \mathrm{~mL}$ of a $39 \mathrm{mM}$ solution of sodium citrate were quickly added to the solution and boiled for $2 \mathrm{~h}$. The ruby-red solution obtained was kept at room temperature and properly diluted before use. Transmission electron microscopy (TEM) images were collected using a JEM-2100F FETEM (JEOL) working at an acceleration voltage of $200 \mathrm{kV}$. Steady-state absorbance and fluorescence measurements were recorded on a Cary 100 spectrophotometer and a Photon Technology International (PTI) fluorimeter, respectively. Raman spectra were recorded in a Horiba Xplora microscope configured with $532 \mathrm{~nm}($ at $24 \mu \mathrm{W})$ and $785 \mathrm{~nm}($ at $50 \mu \mathrm{W})$ laser lines at $100 \%$ power. Data analyses were done using LabSpec 6 software.

Fluorescence spectroscopy measurements: Initial fluorescence testing was run to determine 85 appropriate concentrations of 7-Mercapto-4-methylcoumarin (dimethyl sulfoxide -DMSOsolution) for fluorescence testing. Kinetic studies were performed by monitoring the fluorescence of fixed-volume AuNP solutions ( $12 \mathrm{~nm}$ average diameter, $1.2 \mathrm{nM})$ in the presence of varying amounts of 7-mercapto-4-methylcoumarin; kinetic runs were followed at $430 \mathrm{~nm}$ (Excitation wavelength $=358 \mathrm{~nm}$ ). In addition, fluorescence spectra were recorded by

90 exciting 7-mercapto-4-methylcoumarin solutions before and after $1.5 \mathrm{~h}$ of kinetic testing. 
Surface enhanced Raman spectroscopy measurements: AuNP's surface functionalization was monitored utilizing two different thiols, namely thiophenol (PhSH) and 2Naphthalenethiol (NaphSH). Stock solutions of $\mathrm{PhSH}$ were prepared daily and diluted in purified 18.2 $\mathrm{M} \Omega$ water; while stock solutions of $\mathrm{NaphSH}$ were prepared in EtOH and stored at

$954{ }^{\circ} \mathrm{C}$ for a maximum of one week, followed by the appropriate daily dilution in a 1:1 EtOH/Water mixture. Measurements were performed in solution upon addition of thiol into AuNP $(\sim 60 \mathrm{~nm}$ average diameter, $31 \mathrm{pM}$ ) solutions at given concentrations. SERS measurements were performed on AuNP solution in the presence of various thiol concentrations and recorded at the following acquisition conditions: $785 \mathrm{~nm}$ laser $(25 \%$ power $), 5 \mathrm{~s}$ integration time, 10

100 accumulations per spectrum and $60 \mathrm{~s}$ measurement interval time. The peak areas were calculated using LabSpec software (HORIBA).

All kinetics experiments were fitted using a user-defined fitting with Kaleidagraph or Origin software.

\section{RESULTS AND DISCUSSION}

\section{Kinetics based on fluorescence spectroscopy}

In our work, fluorescence spectroscopy was used as a suitable tool to study different combinations of AuNP and various sensing molecules (Scheme 1). While analyzing the obtained data to extract kinetic and mechanistic information, we use a pragmatic approach by trying to answer the question on hand: how fast can thiols bind to the gold-nanoparticle surface?

110 We have found that 7-mercapto-4-methyl coumarin (C-SH) is an excellent substrate for fluorescence spectroscopy, and commercially available. Interestingly, C-SH is weakly 
fluorescent as a result of non-radiative deactivation (13), attributed to the thione resonance structure contributions (Scheme 2) (11).

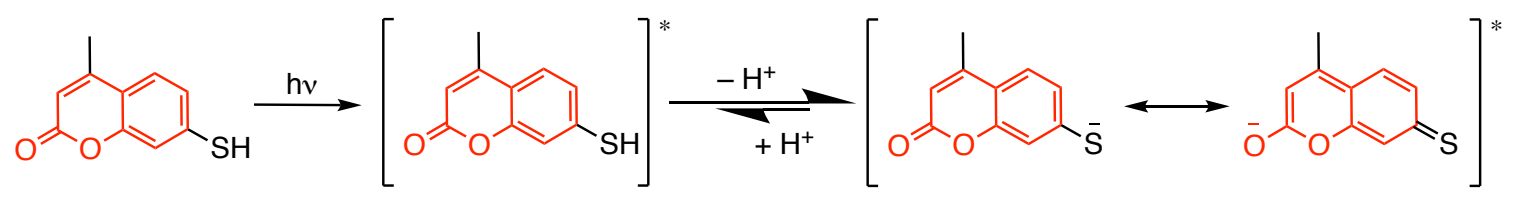

115 Scheme 2. Excited C-SH is prone to deprotonate and favor the thione-like resonance form (nonemissive). Notice in red the coumarin core. Adapted from Ref (11).

When the S-H bond is substituted, for example by a methyl group (C-SMe) the molecule becomes strongly fluorescent (Figure 1), despite what is expected for its hydroxy counterpart

120 (14). Notice that Figure 1 shows that C-SMe is a much stronger emitter than C-SH, typically 20100 times. We have explained this effect in an earlier contribution using Scheme 2 to rationalize the effect (11). Additionally, a modest fluorescence enhancement due to the presence of AuNP is detected, in contrast to typical plasmonic enhancements reported to be an order of magnitude larger (15). It is known that while proximity enhances the signal, surface contact results in

125 emission quenching; the experimental observation depends on the balance of these effects, on the system as well as on the experimental conditions $(15,16)$. 


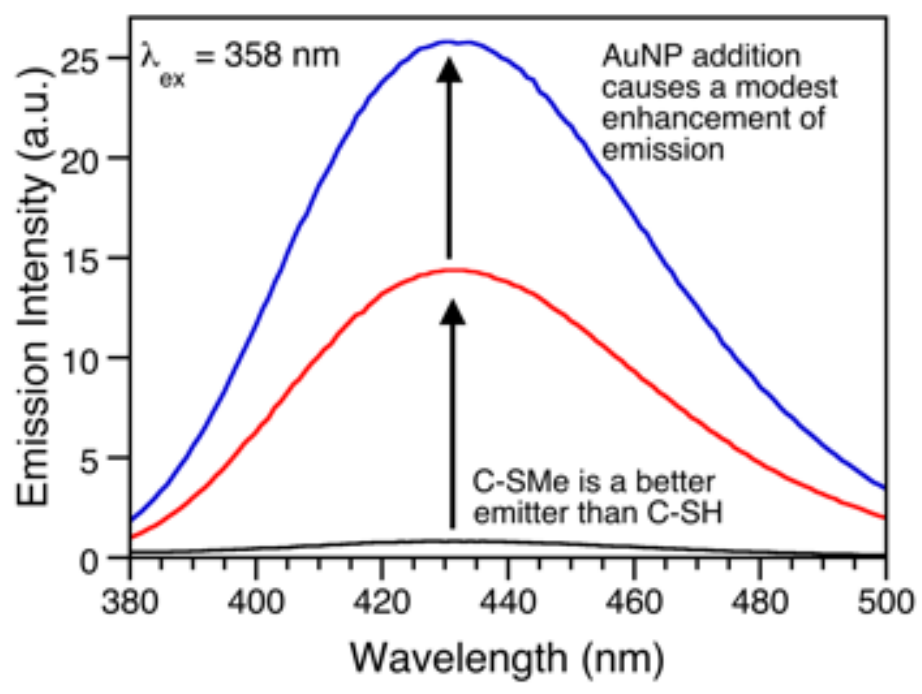

Figure 1. Emission spectra of $0.52 \mu \mathrm{M}$ of C-SH (black), $0.052 \mu \mathrm{M}$ of C-SMe (red) and the C-SMe sample after addition of $1.2 \mathrm{nM}$ AuNP (blue) and a 5 min incubation. All in 10\% DMSO: $\mathrm{H}_{2} \mathrm{O}$.

The addition of C-SH to the gold surface leads to the displacement of citrate and the formation of thiolate bonds (12), Scheme 3, with concomitant spectral changes in the AuNP region ( $\sim 530$ $\mathrm{nm}$ ) and the appearance of the coumarin absorption band in the $360 \mathrm{~nm}$ region, Figure 2. Thus, the S-H bond can be eliminated by coordination to the gold surface (Scheme 3), which is

135 typically the key step in AuNP derivatization. With this in mind, we decided to design a simple way to determine how fast the interaction between thiols and AuNP surface can take place, using $\mathrm{C}-\mathrm{SH}$ as a probe. Thus, we expect the thione-like resonance form of C-SH to be less favored as the thiol moiety is engaged in binding to the AuNPs surface, increasing the fluorescence emission of the coumarin. The increase in fluorescence signal can account then for the formal

140 interaction between the thiol moiety and the Au surface, and help reveal kinetic information about the process. While fluorescence quenching is expected, when fluorophores sit right on the surface of plasmonic materials such as AuNP, the experimental balance in this case is a moderate (but readily detectable) fluorescence enhancement (16). Thus, the use of fluorescence 
enhancement provides a novel approach to monitor reactivity with the gold plasmonic surface.

145 The changes in Figure 2 are attributed to modifications on the surface of the AuNPs, as well as changes in the dielectric media, as a consequence of the presence of C-SH. Changes produced by the addition of the different solvent were ruled out. Pure DMSO was added to AuNP under the same conditions and no deviations in the absorption spectrum were detected.

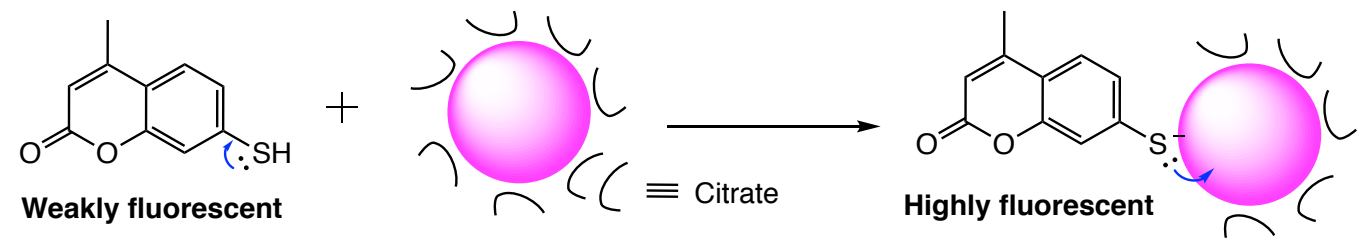

150 Scheme 3. The formation of strong thiolate bonds causes the displacement of citrate from the gold surface.

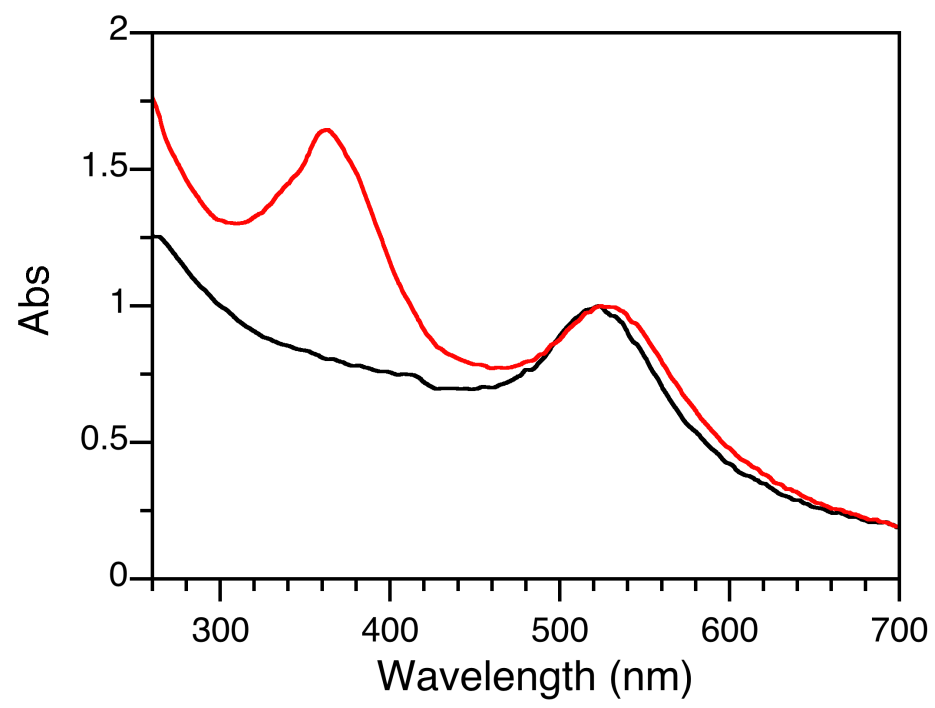

Figure 2. Normalized absorption spectra of AuNPs in the absence (black) and in the presence (red) of $160 \mu \mathrm{M}$ of C-SH. Notice the plasmon band maximum for AuNP shifts from $522 \mathrm{~nm}$ to $528 \mathrm{~nm}$.

AuNP used for fluorescence spectroscopy (Figure 3) have an average size of $12 \mathrm{~nm}$, significantly smaller than those used for Raman spectroscopy ( $\sim 60 \mathrm{~nm}$, vide infra). Particles were initially tested at two different AuNP concentrations: 2.4 and $1.2 \mathrm{nM}$ (calculated as previously reported 
(16)). Figure 4 shows how the emission at $440 \mathrm{~nm}$, corresponding to C-SH, increases after

160 mixing with AuNP solution. Notice that while essentially the same rate constants are obtained, diluted AuNP solutions reach higher fluorescence intensities, more likely due to better light penetration and minimal light re-absorption.

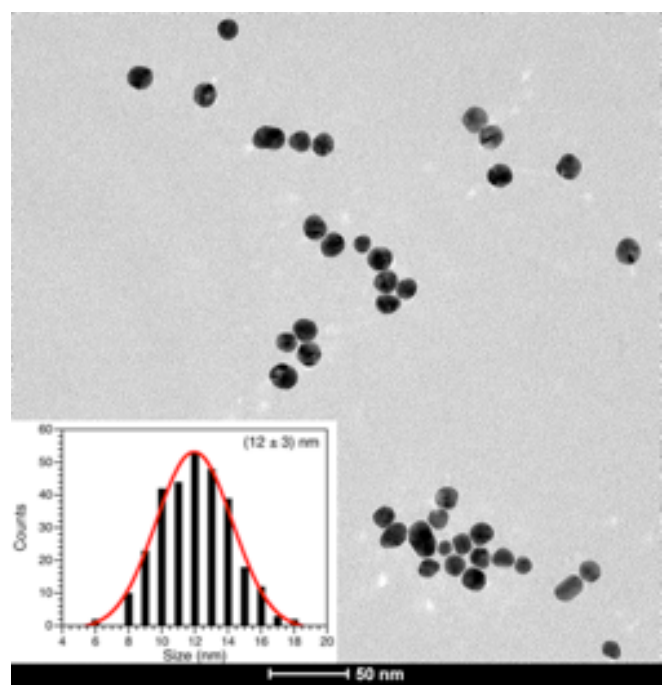

165

Figure 3. HR-TEM images of the AuNP used for fluorescence experiments and (inset) particle size distribution for one of the two batches on AuNP used.

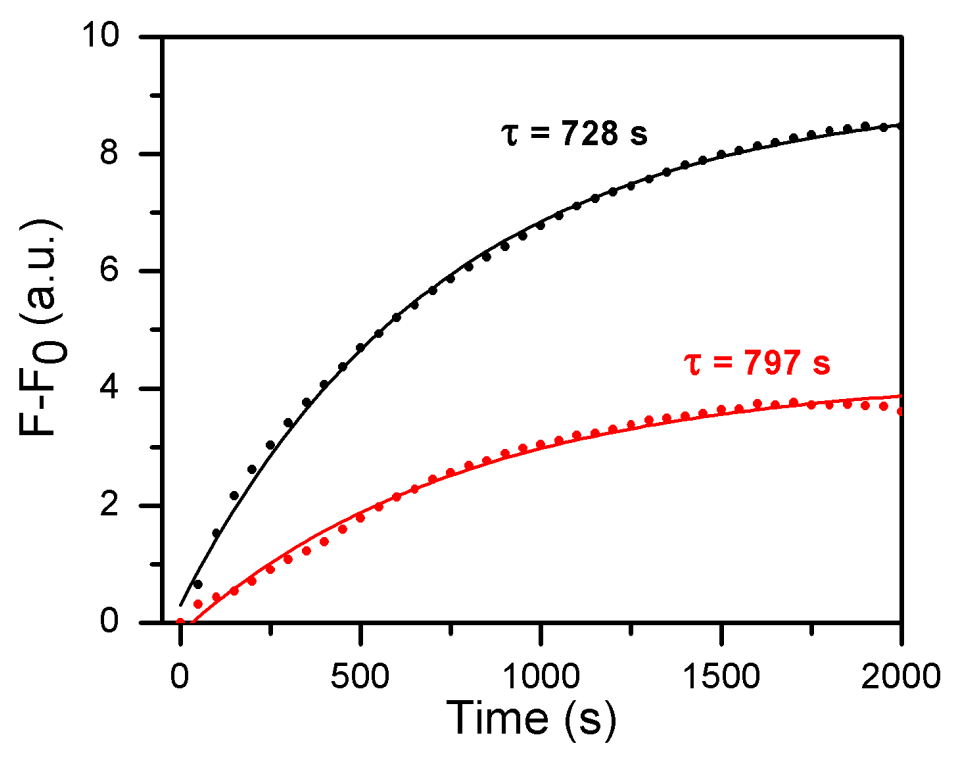


170 Figure 4 . Fluorescence intensity change $\left(\mathrm{F}-\mathrm{F}_{0}\right)$ monitored at $430 \mathrm{~nm}\left(\boldsymbol{\lambda}_{\mathrm{ex}}=358 \mathrm{~nm}\right)$ versus time for $4 \mu \mathrm{M}$ of C-SH in the presence of $1.2 \mathrm{nM}$ (black) and $2.4 \mathrm{nM}$ (red) of AuNP. Fit to a first-order exponential growth model (solid line). Notice same rate constant values, kobs, are obtained for both NP concentrations.

Figure 4 clearly shows that changes in the AuNP concentration led to unusual effects (such as

175 more signal with less AuNP) that while readily explained on a qualitative basis pose serious challenges for quantification. Thus, most of the experiments that follow center on kinetic studies where thiol concentrations are changed while maintaining the AuNP concentration constant. Representative results are shown in Figure 5, where C-SH was added in concentrations ranging from 1 to $6 \mu \mathrm{M}$ and monitored for $2000 \mathrm{~s}$. Note that only the emission growth component of the

180 signal is displayed in Figure 5, as C-SH, while weak is somewhat emissive (see Figure 1). This emission is probably slightly enhanced by non-reactive initial interaction with the gold surface. An example of the uncorrected traces is shown as an inset in Figure 5. A simple visual inspection of Figure 5 shows that about 30 minutes is required for $90 \%$ of the reaction to take place.

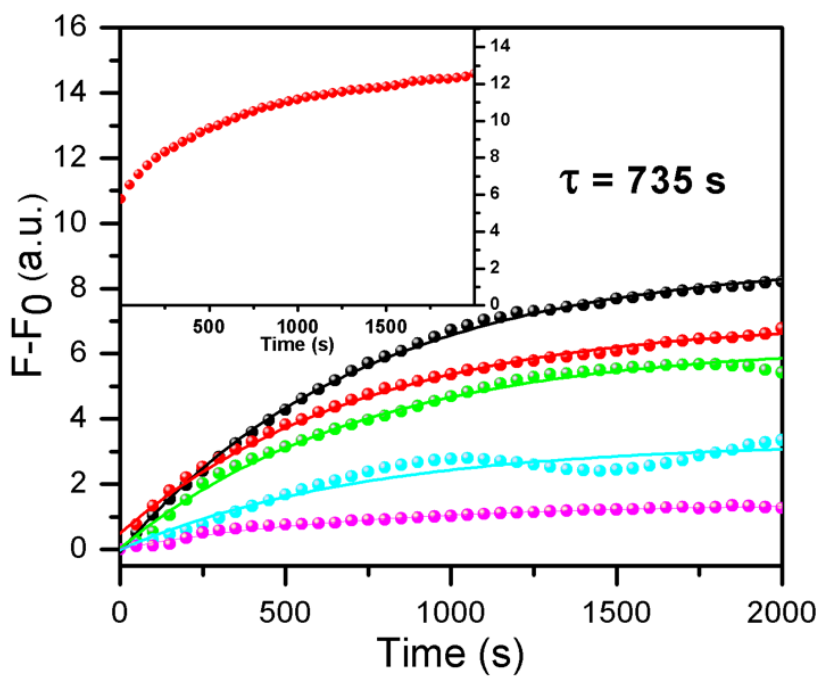

185 Figure 5. Fluorescence intensity for different concentrations of C-SH added to $1.2 \mathrm{nM}$ of AuNP and monitored at $430 \mathrm{~nm}$ versus time. The fitting lines shown have been calculated by global analysis of the reaction kinetics. Concentration of C-SH from the bottom to the top: 1, 2, 3, 4, $6 \mu \mathrm{M}$. Inset: uncorrected trace for $4 \mu \mathrm{M}$ solution. 
The surface of each AuNP must present sites with different reactivity, further, the material size

190 distribution is not monodisperse, as Figure 3 shows. Under these conditions, it is remarkable that the plots of Figure 5 can be reasonably fitted with a monoexponential growth, in other words, they follow excellent Langmuir-type first order kinetics. In fact, it was possible to use a kinetic global fitting approach to fit the data in Figure 5 using equation (1).

$$
\mathrm{A}=\mathrm{A}_{0}\left(1-\mathrm{e}^{-k \mathrm{t}}\right)
$$

195 where $k$ is the observed rate constant for the growth of A (enhanced Raman or emission signal) and is equal to the inverse of the lifetime $(1 / \tau)$. We obtain $k$ as the value that minimizes the mean squared error between the fitted and the observed kinetic curves with different values of $A_{0}$.

Global kinetic analysis of Figure 5 yields a lifetime $(\tau)$ of 735 s. Considering that three lifetimes corresponds to $95 \%$ completion, the visual estimate of 30 minutes mentioned above 200 appears quite reasonable. Beyond a single lifetime or rate constant, global analysis also yields a projected plateau value for the signal $\left(A_{0}\right)$. These values were recorded and plotted against concentration in Figure 6. The plot reaches a plateau that implies the mercaptan and not the AuNP is the likely limiting reagent in this system. At the highest C-SH concentration, this corresponds to $\sim 3300$ thiolate bonds per nanoparticle. 


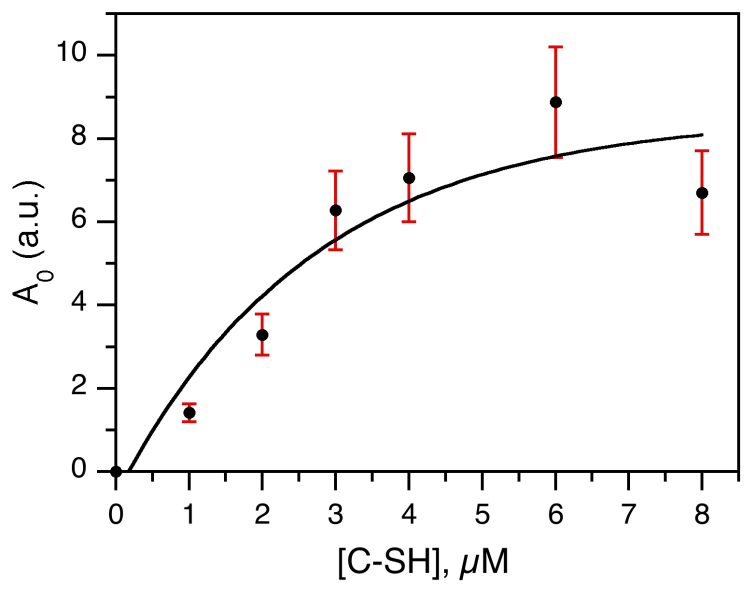

Figure 6. Fluorescence intensity change $\left(\mathrm{A}_{0}\right)$ determined from the calculated plateau in figure 5 plotted as a function of the $\mathrm{C}-\mathrm{SH}$ concentration. Errors estimated as $15 \%$.

In order to prove the reproducibility of this methodology, a second batch of AuNP $(\sim 17 \mathrm{~nm}$, see

210 SI) was prepared and the same type of kinetics were evaluated. Figure 7 shows the kinetic traces obtained.

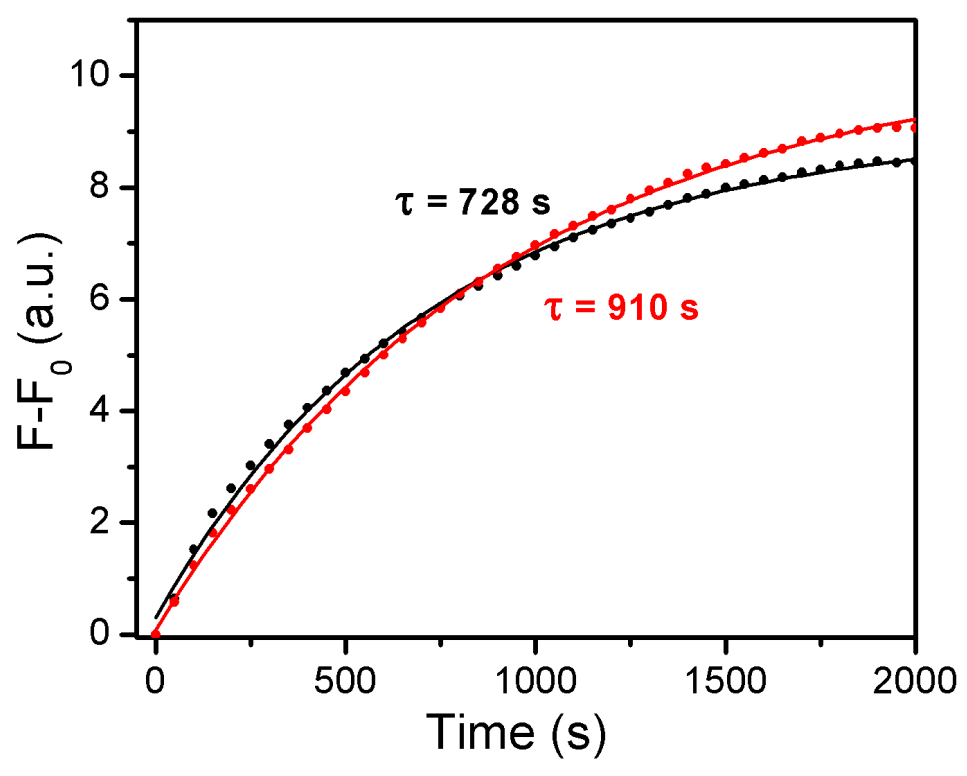

Figure 7. Fluorescence intensity for $4 \mu \mathrm{M}$ of C-SH added to $1.2 \mathrm{nM}$ of AuNP batch 1 (black) and batch 2 (red) and monitored at $430 \mathrm{~nm}$ versus time. The fitting lines shown have been calculated by first exponential growth kinetics 215 (eq. 1). 
The lifetime values of 767, 638 and $660 \mathrm{~s}$, derived from single curve analysis (Figure 7) and global analysis (Figure 5) illustrate the type of reproducibility that can be expected in these systems as the sample batch and/or type of analysis is changed.

\section{Kinetics based on Raman spectroscopy}

Surface enhanced Raman spectroscopy (SERS) is also an important and complementary tool in determining the reactivity of the S-H bond towards the gold surface. The best suited molecules for SERS differ from those preferred for fluorescent studies, and thus, non-fluorescent or weakly

225 fluorescent aromatic molecules, such as PhSH and NaphSH (see Scheme 1) were the choices for these studies. AuNP concentration was also optimized in order to have greater Raman signal enhancement. Further, larger particles are optimal for SERS, and thus citrate-covered $65 \mathrm{~nm}$ AuNP were preferred. The Raman spectra for PhSH and NaphSH are shown in Figure 8; notice in the inset that in addition to polydispersity, the sample shows some polymorphism. 


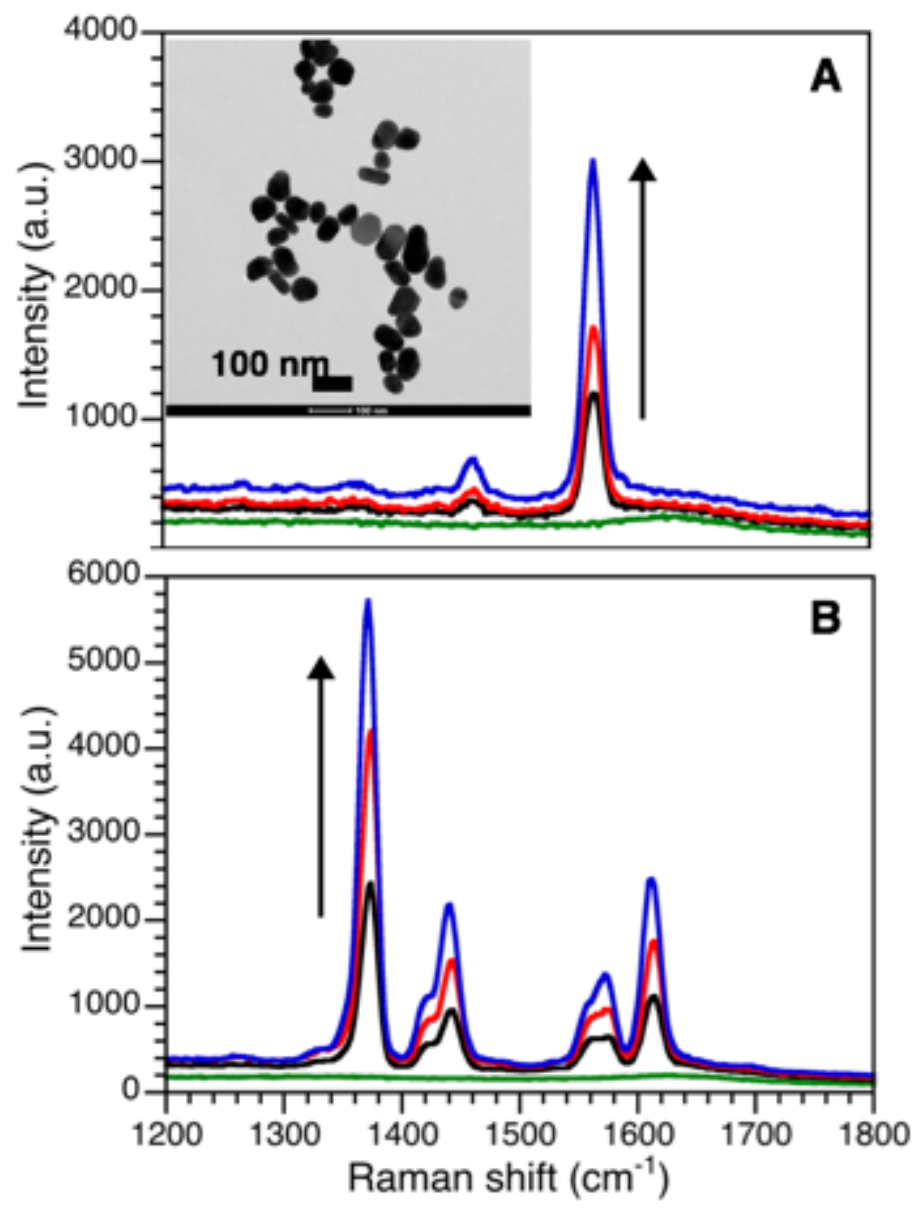

Figure 8. Raman spectra for thiol-modified AuNP obtained with different concentrations of thiol after $30 \mathrm{~min}$ of mixing. (A): PhSH at 0 (green), $60 \mathrm{nM}$ (black), $100 \mathrm{nM}$ (red) and $200 \mathrm{nM}$ (blue). (B): NaphSH at 0 (green), 50 $\mathrm{nM}$ (black), $100 \mathrm{nM}$ (red) and $200 \mathrm{nM}$ (blue). In both cases, the Raman signal increases with concentration

235 In the case of $\mathrm{PhSH}$, Raman peaks at $408(\mathrm{C}-\mathrm{S}), 1067(\mathrm{C}-\mathrm{H})$ and $1565 \mathrm{~cm}^{-1}(\mathrm{C}=\mathrm{C})$ could be monitored to observe the enhancement that results from binding at the AuNP surface. In our case, we found the $1565 \mathrm{~cm}^{-1}$ peak to be the most convenient for PhSH (Figure 9). Likewise, we followed the addition of NaphSH monitoring the area of the $\mathrm{C}=\mathrm{C}$ peak at $1370 \mathrm{~cm}^{-1}$ (Figure 10). 


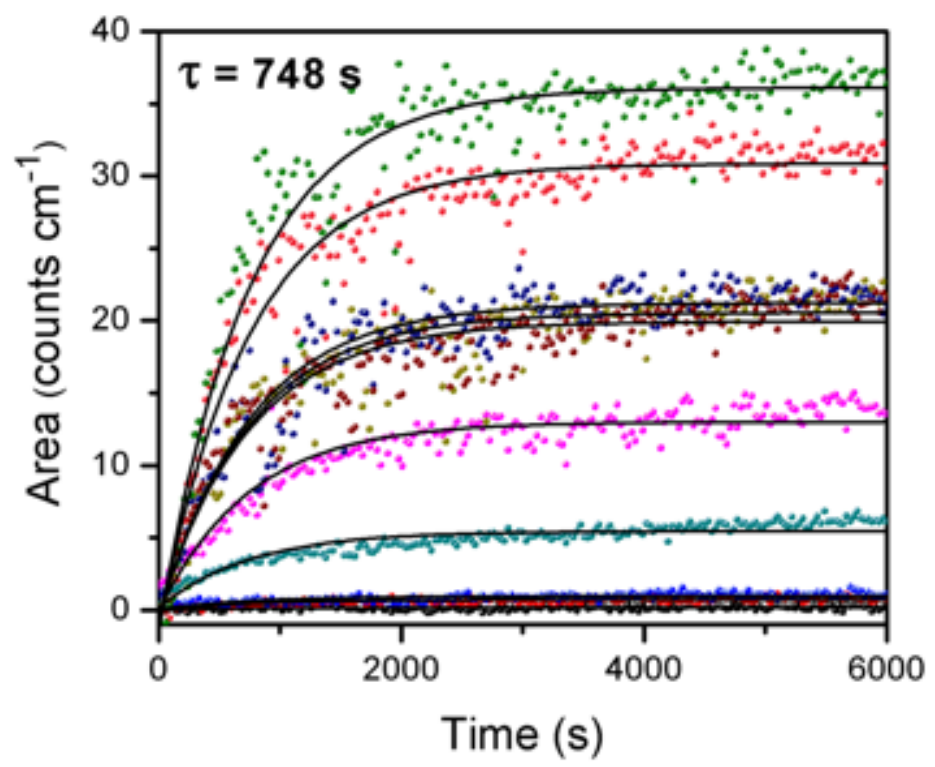

240 Figure 9. Kinetics experiment for the AuNP surface modification with different concentrations of $\mathrm{PhSH}$ in water added to $31 \mathrm{pM}$ AuNP solution, monitoring the area of the $\mathrm{C}=\mathrm{C}$ peak at $1565 \mathrm{~cm}^{-1}$ and fitted using global analysis. $[\mathrm{PhSH}]$ from the bottom to the top: 20, 40, 50, 55, 60, 80, 100, 140, 200, $220 \mathrm{nM}$.

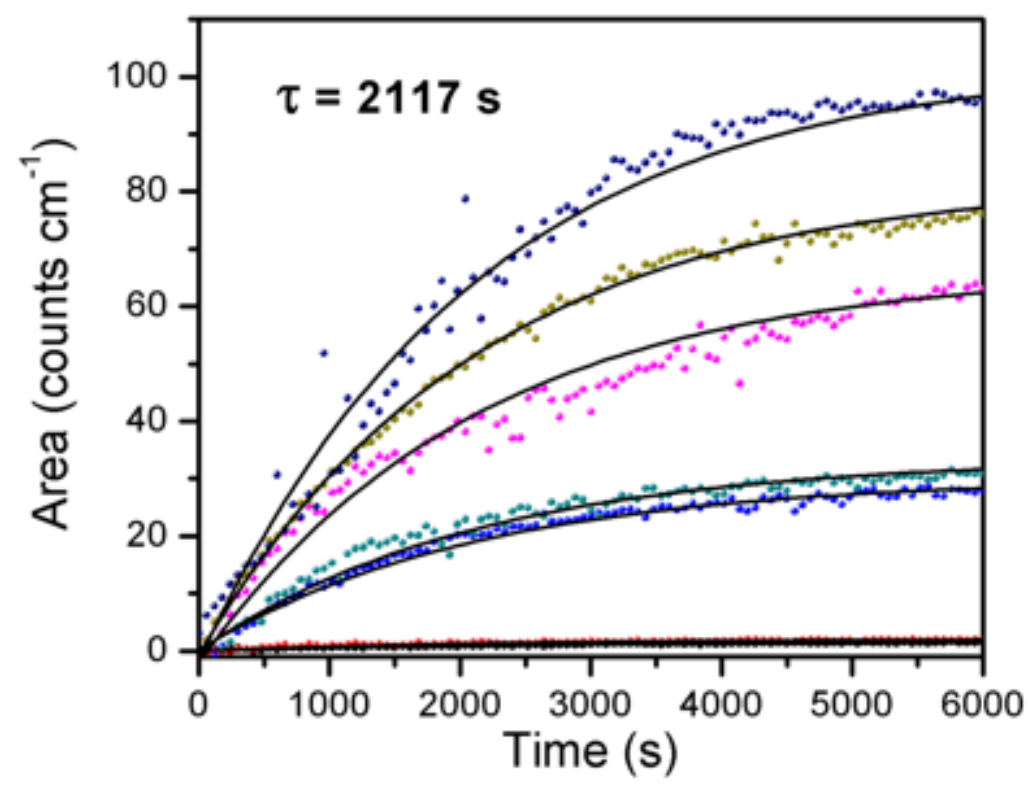

245 Figure 10. Kinetics experiment for the AuNP surface modification with different concentrations of NaphSH in water added to $31 \mathrm{pM}$ AuNP solution, monitoring the area of the $\mathrm{C}=\mathrm{C}$ peak at $1370 \mathrm{~cm}^{-1}$ and fitted using global analysis. [NaphSH] from the bottom to the top: 20, 40, 50, 60, 90, 100, $200 \mathrm{nM}$. 

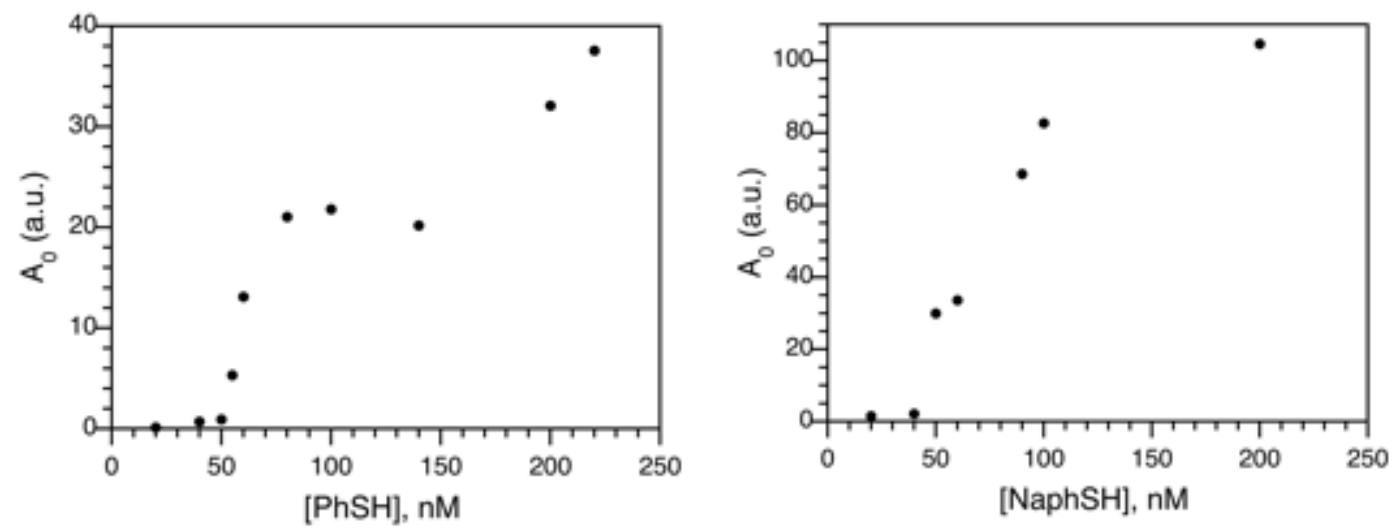

Figure 11. Raman area change $\left(\mathrm{A}_{0}\right)$ determined from the calculated plateau in figure 8 and 9 plotted as a function 250 of the thiol concentration. Notice the saturation of the plot up to $\sim 3200$ molecules of thiol per AuNP, corresponding to a concentration of $100 \mathrm{nM}$ of thiol per $31 \mathrm{pM}$ AuNP solution.

\section{Competition binding by lipoic acid}

The case of lipoic acid (LA) is particularly interesting, as its behavior should mimic disulfides,

255 however -due to its cyclic structure- once LA binds to the gold surface produces more stable molecular arrangements because of the formation of two S-Au bonds. (17). We have designed two types of fluorescence experiments where LA and C-SH compete for the gold surface. In the first one equimolar concentrations of both are added simultaneously to a AuNP solution. As shown in Figure 12, the presence of LA has virtually no effect on the growth of the fluorescence

260 of C-SH from the gold-bonded mercaptocoumarin. This result implies that for C-SH and LA, thiols are more reactive than disulfides, likely because S-S bond breaking is required for LA. In the second type of experiments the reagents are exactly the same, however, in this case the AuNP were pre-incubated at the same concentration of LA for $24 \mathrm{~h}$, prior to addition of C-SH. These results are also presented in Figure 12, and show a smaller and somewhat slower fluorescence growth, illustrating that LA has bonded to the gold surface thus reducing the number of active sites available for C-SH reaction. 


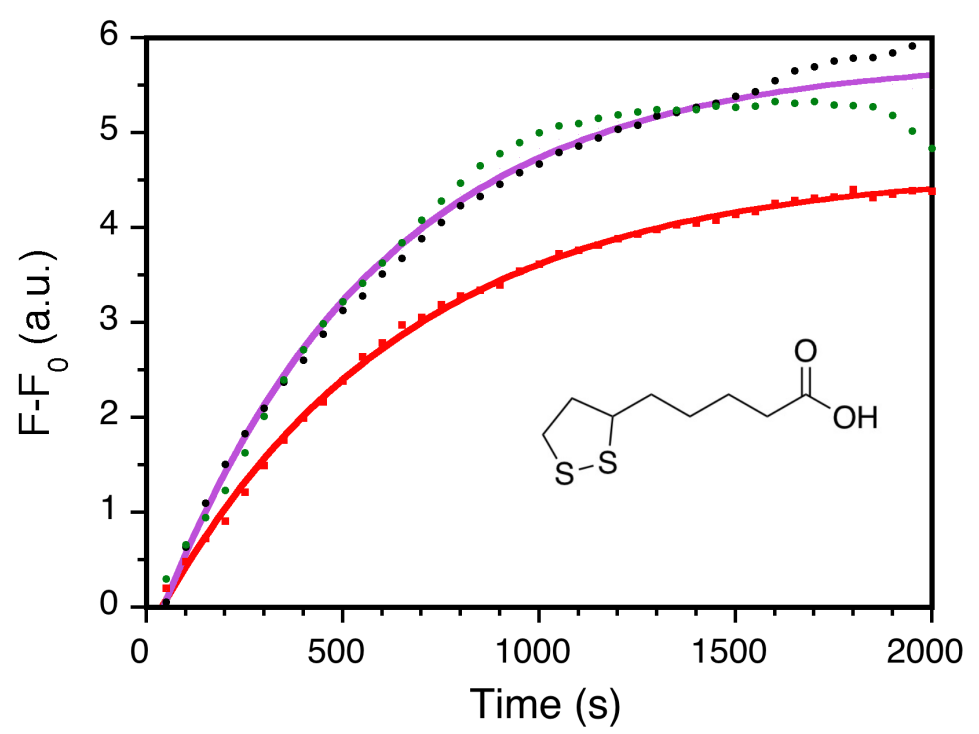

Figure 12. Fluorescence intensity change $\left(\mathrm{F}-\mathrm{F}_{0}\right)$ monitored at $430 \mathrm{~nm}$ versus time from solution of $1.2 \mathrm{nM}$ of AuNP after addition of $4 \mu \mathrm{M} \mathrm{C}$-SH (black), $4 \mu \mathrm{M} \mathrm{C}$-SH and $4 \mu \mathrm{M} \mathrm{LA}$ (green) and

$2704 \mu \mathrm{M}$ C-SH added to a AuNP solution previously incubated for $24 \mathrm{~h}$ with $4 \mu \mathrm{M}$ LA (red). The purple line fits simultaneously black and green data (differences within the experimental error).

Thus, the competition of C-SH with LA shows that thiols are far more reactive than disulfide, as adding LA as a competitive reagent does not affect the growth curve giving a growth lifetime 275 of $560 \pm 70 \mathrm{~s}$. On the other hand, if AuNP are pre-incubated overnight with LA, the disulfide can bind to the surface (frequently described as two-footed binding) and protect it from the thiols attack. This results in a lifetime of $620 \pm 70 \mathrm{~s}$ and the final fluorescence is only $78 \%$ of that achieved without pre-incubation. With pre-incubation less sites are available, but those that remain available are within experimental errors just as reactive as those encountered on a fresh

280 surface. Overall LA binding must be about ten times slower than thiol reaction, thus needing overnight incubation to make a real difference in C-SH binding.

\section{CONCLUSION}


These results prove the reactivity of several thiols occurs within the same timescale, however

285 disulfides (such as LA) react much more slowly. To answer the title question, we recommend one to two hour wait for thiol binding to be essentially complete, while for disulfides, overnight incubation is recommended. Any "ready-mix" strategy is bound to lead to results obtained while the formation of thiolate bonds was still in progress.

\section{ACKNOWLEDGMENTS}

Thanks are due to the Discovery program from Natural Sciences and Engineering Research Council of Canada, the Canada Research Chairs Program, the Canada Foundation for Innovation and the Canadian Genome Program.

\section{SUPPLEMENTARY MATERIALS}

295 Supporting materials include details of the calculation of nanoparticle concentrations and additional TEM images.

\section{REFERENCES}

1. Hakkinen, H. (2012) The gold-sulfur interface at the nanoscale. Nat. Chem. 4, 443-455. 2. Pensa, E., E. Cortes, G. Corthey, P. Carro, C. Vericat, M. H. Fonticelli, G. Benitez, A. A. 300 Rubert and R. C. Salvarezza (2012) The Chemistry of the Sulfur-Gold Interface: In Search of a Unified Model. Acc. Chem. Res. 45, 1183-1192.

3. Goldmann, C., R. Lazzari, X. Paquez, C. Boissiere, F. Ribot, C. Sanchez, C. Chaneac and D. Portehault (2015) Charge Transfer at Hybrid Interfaces: Plasmonics of Aromatic Thiol-Capped Gold Nanoparticles. ACS Nano 9, 7572-7582.

305 4. Reimers, J. R., M. J. Ford, S. M. Marcuccio, J. Ulstrup and N. S. Hush (2017) Competition of van der Waals and chemical forces on gold-sulfur surfaces and nanoparticles. Nat. Rev. Chem. 1, 0017. 
5. Stamplecoskie, K. G., G. Yousefalizadeh, L. Gozdzialski and H. Ramsay (2018) Photovoltaics as an Experimental Tool for Determining Frontier Orbital Energies and Photocatalytic Activity of Thiol Protected Gold Clusters. J Phys. Chem. C 122, 1373813744.

6. Yousefalizadeh, G. and K. G. Stamplecoskie (2018) Norrish type I photochemistry as a powerful tool in the isolation of thiol protected Au25SR18 clusters. J. Photochem. Photobiol., A 353, 251-254.

315 7. Villarreal, E., G. F. G. Li, Q. F. Zhang, X. Q. Fu and H. Wang (2017) Nanoscale Surface Curvature Effects on Ligand-Nanoparticle Interactions: A Plasmon-Enhanced Spectroscopic Study of Thiolated Ligand Adsorption, Desorption, and Exchange on Gold Nanoparticles. Nano Lett. 17, 4443-4452.

8. DeVetter, B. M., P. Mukherjee, C. J. Murphy and R. Bhargava (2015) Measuring binding kinetics of aromatic thiolated molecules with nanoparticles via surface-enhanced Raman spectroscopy. Nanoscale 7, 8766-8775.

9. Stamplecoskie, K. G., J. C. Scaiano, V. S. Tiwari and H. Anis (2011) Optimal Size of Silver Nanoparticles for Surface-Enhanced Raman Spectroscopy. J Phys. Chem. C 115, 14031409.

325 10. Ansar, S. M. and C. L. Kitchens (2016) Impact of Gold Nanoparticle Stabilizing Ligands on the Colloidal Catalytic Reduction of 4-Nitrophenol. ACS Catal. 6, 5553-5560.

11. Lanterna, A. E., M. Gonzalez-Bejar, M. Frenette and J. C. Scaiano (2017) Photophysics of 7-mercapto-4-methylcoumarin and derivatives: complementary fluorescence behaviour to 7-hydroxycoumarins. Photochem. Photobiol. Sci. 16, 1284-1289.

330 12. Vanegas, J. P., J. C. Scaiano and A. E. Lanterna (2017) Thiol-Stabilized Gold Nanoparticles: New Ways To Displace Thiol Layers Using Yttrium or Lanthanide Chlorides. Langmuir 33, 12149-12154.

13. Gonzalez-Bejar, M., M. Frenette, L. Jorge and J. C. Scaiano (2009) 7-Mercapto-4methylcoumarin as a reporter of thiol binding to the CdSe quantum dot surface. Chem.

335 Commun., 3202-3204.

14. Demelo, J. S. S., R. S. Becker and A. L. Macanita (1994) Photophysical Behavior of Coumarins as a Function of Substitution and Solvent - Experimental-Evidence for the Existence of a Lowest Lying ${ }^{1}\left(\mathrm{n}, \pi^{*}\right)$ State. J Phys. Chem. 98, 6054-6058.

15. Anger, P., P. Bharadwaj and L. Novotny (2006) Enhancement and quenching of singlemolecule fluorescence. Phys. Rev. Lett. 96.

16. Pacioni, N. L., M. Gonzalez-Bejar, E. Alarcon, K. L. McGilvray and J. C. Scaiano (2010) Surface Plasmons Control the Dynamics of Excited Triplet States in the Presence of Gold Nanoparticles. J. Am. Chem. Soc. 132, 6298-6299.

345 from gold nanoparticles. Chem. Commun. 49, 5639-5641. 